Article

\title{
A Robot System Maintained with Small Scale Distributed Energy Sources
}

\author{
Jaehyun Kim and Chanwoo Moon * \\ School of Electrical Engineering, Kookmin University, Seoul 02707, Korea; goss818@gmail.com \\ * Correspondence: mcwnt@kookmin.ac.kr; Tel.: +82-2910-4779
}

Received: 10 September 2019; Accepted: 5 October 2019; Published: 11 October 2019

check for updates

\begin{abstract}
An energy autonomy system is sustained by energy from independent and distributed sources. This paper presents a robot system that obtains energy from renewable energy sources distributed over a large area with limited storage capacity. We constructed a linearized charge model to estimate the required energy node capacity and distribution for the robot to survive. For a robot to obtain energy from an energy source, it must be able to recognize the energy node and able to receive energy reliably. We used wireless power transfer to solve conventional contact charging problems, such as mechanical complexity and unstable contact, and image information was used to recognize the energy nodes and align the transmission coils accurately. A small scale renewable energy source was constructed and a charge experiment was conducted to verify the proposed autonomy system feasibility.
\end{abstract}

Keywords: robot energy autonomy; renewable energy; charge model; wireless power transfer; image information

\section{Introduction}

Mobile robots and drones have found wide applications across many industries, including agriculture, delivery, search, and exploration. Some mobile robots incorporate combustion engines and fuel tanks, but most are operated by electric motors and powered by an on-board battery, which requires periodic recharging as it becomes exhausted. Battery recharging has been an acknowledged problem for some time. The most common method is to manually charge the battery. Home robots, particularly vacuum cleaning devices, generally recharge automatically using various systems, with the robot finding the recharge station using vision [1] or infrared LEDs [2]. Other automatic charging methods have been proposed to allow robots to intake power or replace a charged battery autonomously $[3,4]$. Such methods offer a reduced time required for charging, but current mechanisms are complicated and unreliable. Energy sources used to charge robots are usually commercial power sources, but various energy sources for mobile robots have also been considered. A typical example is photovoltaic cells, which are widely used, but difficult to use in the absence of sunlight or when there are payload limitations, e.g., rotary wing drones. A robot that consumes organic substances as its power supply rather than electrical energy has been proposed [5], as well as others that harvest their own energy. However, the latter option only applies for micro robots, e.g., insect-sized robots, because the amount of available energy is relatively small [6].

Energy autonomy systems are sustained by energy from independent and distributed energy sources [7]. This paper presents an energy autonomy system in which a robot obtains energy from a distributed independent external energy sources with limited storage capacity, such as renewable energy, in the event that the robot does not receive sufficient energy from conventional commercial power sources. Currently, robotic energy autonomy studies have only been conceptual [8]. Khonji et al., proposed a drone charging system using wireless power transmission from a solar generator large 
enough to charge the robot directly without storing energy. However, they did not consider the energy balance between the robot and the energy source [9].

To realize energy autonomy, the robot must be able to recognize the distributed energy sources itself, which come in a wide range of types and shapes, and be able to successfully transfer energy from the source to the robot. Conventional automatic charging methods employ mechanical contact, which is an effective commercial technology. Mobile robots generally do not have sufficient positional accuracy to make unassisted contact with a recharge station, and hence guide mechanisms around the recharge station are used to correct robot position and ensure stable contact with the recharge station $[1,2]$. However, electrical contact is often incomplete in practical outdoor environments, where the charging terminal can be contaminated by dust, moisture, abrasion, etc. Battery exchange type recharging stations are difficult to use as distributed small scale energy sources because the exchange mechanisms are somewhat complicated. Therefore, we adopted wireless power transmission energy transfer to address conventional energy transfer problems. Wireless charging has previously been regarded as an auxiliary method due to low efficiency and environmental problems, but has developed significantly recently to provide a solid charging method option [10]. In the field of mobile communication, much research on wireless power transfer technology has been reported. Ju et al. have proposed a system that enables mobile devices to harvest energy and transmit information through Hybrid APs (Access Points) [11] and Vamvakas et al., proposed a system for supplying energy to mobile devices through power stations [12]. Several studies have considered wireless power transfer for mobile robots, proposing automatic charging systems for mobile robots using image information [13,14]. A power station was practically implemented for automatic charging rotary wing drones [15]. A robot supplied energy to the sensor network [16] and fellow robots [17]. Although wireless power transfer simplifies the required connection mechanisms, transmission efficiency remains relatively low and recharging is time consuming. Hence only a small portion of the energy produced can be used by robots and the proportion of time spent charging is high compared to total activity time. Therefore, it is essential to analyze whether the robot has enough energy and time to work.

This study proposes an autonomous robotic system that can survive by acquiring energy from distributed sources with limited storage capacity, similar to the honey bee behavior. The distributed energy sources are small-scale, collecting energy in a storage device that is delivered to the robot. Previous studies considered energy harvesting robots [6], where the energy harvesting device was inside the robot. In contrast, this study proposes small-scale renewable energy sources outside the robot, and the robot is retained by acquiring this energy. We constructed a linear charge model with variables for energy node distribution and capacity, and analyzed whether the robot could survive. We adopted an induction-type wireless power transfer to solve contact charging problems. A small-scale thermoelectric energy source was constructed and charging experiments was conducted to verify proposed energy autonomy system feasibility.

The remainder of this paper is organized as follows. Section 2 presents the problem statement and subsequent autonomous energy charge model. Section 3 provides numerical evaluations for the proposed system, and presents experimental results. Section 4 summarizes the paper.

\section{Methodology}

\subsection{Problem Statement}

Figure 1 shows a typical robot activity space, comprising work and energy acquisition areas, with $\mathrm{N}$ energy nodes in the energy acquisition area. When the robot energy is close to exhausted, it visits the energy nodes to recharge its energy. 


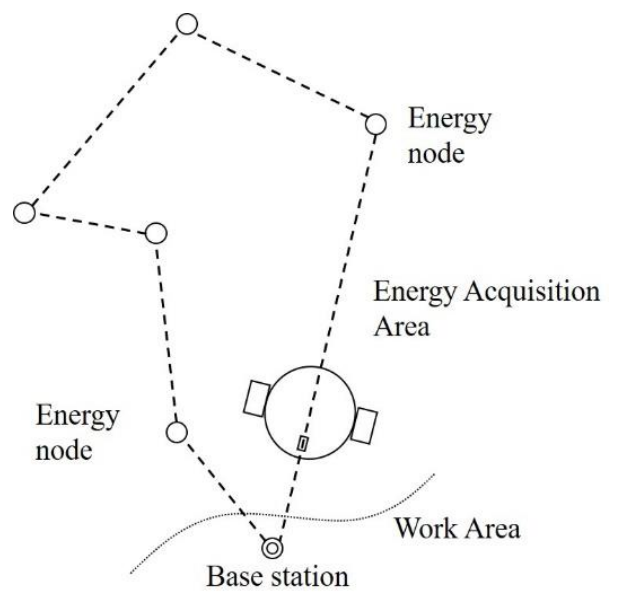

Figure 1. Robot activity space.

Each energy node stores electric energy generated from some source in energy storage (ES), comprising a battery or super-capacitor with limited capacity, and transmits energy to the robot when it visits. Figure 2 shows that each energy node includes an energy source, power converter, energy storage system, and transmitter and coil for power transmission.

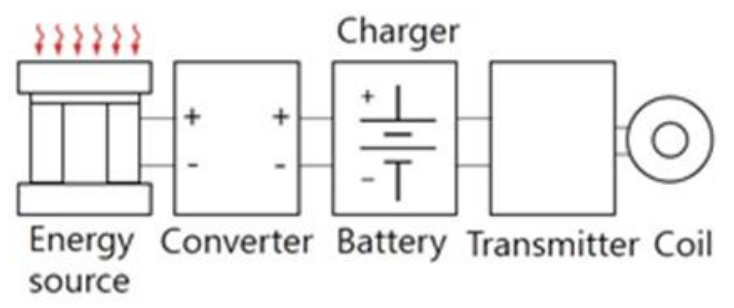

Figure 2. Typical energy node structure.

\subsection{Charge Cycle Model}

The amount of energy to be generated and transferred are approximated using a linear model with the amount of energy produced being proportional to time and can saturate the available storage capacity, and the amount delivered using wireless energy transfer is also proportional to delivery time as Equations (1)-(3),

$$
\begin{gathered}
\epsilon_{i}=\operatorname{sat}_{E_{i}}\left(g_{i} \cdot t_{g}+\epsilon_{i 0}\right), \\
e_{i T}=\mu \cdot t_{T},
\end{gathered}
$$

and

$$
e_{i R}=\eta \cdot \mu \cdot t_{T}
$$

where $\epsilon_{i}$ is stored energy (initially, $\epsilon_{i 0}$ ), $g_{i}$ is energy generation rate, $E_{i}$ is energy storage capacity, $e_{i T}$ is energy transmitted from, and $e_{i R}$ is energy received from energy node $i$ on the robot side; $t_{g}$ is energy generation duration, $\mu$ is wireless power transfer coil energy transfer rate, $\eta$ is energy transfer efficiency, $t_{T}$ is transmission duration, and

$$
\begin{gathered}
\operatorname{sat}_{L}(x)=x, \text { if } x<L \\
=L, \text { if } x \geq L .
\end{gathered}
$$

Since energy the transfer efficiency is $<1$, only a fraction of the produced energy is used in the robot. Energy transfer efficiency is the product of wireless power transfer efficiency, $\eta_{1}$, and robot battery energy efficiency, $\eta_{2}$, i.e., $\eta=\eta_{1} \cdot \eta_{2}$. Wireless power transfer efficiency depends on how well the transmission and receiving coils are aligned, and we determined experimentally that $\eta_{1}<0.8$ for 
induction-type systems. Battery energy efficiency is the ratio of the usable energy to energy received and $\eta_{2} \approx 0.8$ for Li-ion batteries, but can be significantly lower for other battery types [18].

The robot consumes energy to visit an energy node, and the energy it receives must exceed the energy consumed to acquire it. Robot power drain during movement, $p_{\text {trip }}$, consists of power to drive and power consumed by the controller [19],

$$
p_{\text {trip }}=p_{m}+p_{c}
$$

where $p_{m}$ is robot power consumption while driving, i.e., by motor(s) and sensors used for navigation, etc., and $p_{c}$ is power consumption for control, consumed by the controller board.

Work and charge constitute one cycle. Some robots have a sleep function. Power consumption in the sleep state, $p_{s}$, is the minimum to maintain the system, and hence significantly less than consumed in normal operation. The robot can go into a sleep state if energy node production is insufficient. Figure 3 shows a typical robot system operation-charge cycle.

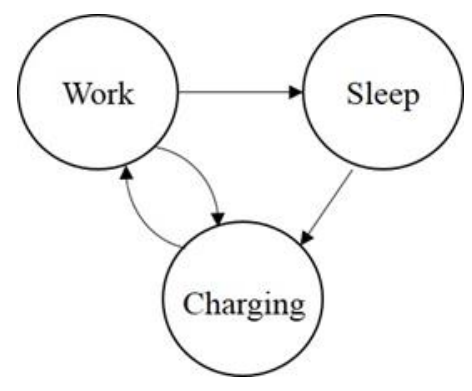

Figure 3. Typical robot operation-charge cycle.

Table 1 shows the symbols used in the charge model.

Table 1. Symbols used in the charge model.

\begin{tabular}{cc}
\hline Symbol & Explanation \\
\hline$g_{\text {tot }}$ & Total energy generation rate for all nodes, $\mathrm{W}$ \\
$\mu$ & Energy transfer rate of wireless power transfer coil, $\mathrm{W}$ \\
$\eta$ & Energy transfer efficiency \\
$p_{c}$ & Robot power consumption for computing, $\mathrm{W}$ \\
$p_{m}$ & Robot power consumption for driving, $\mathrm{W}$ \\
$p_{w}$ & Average robot power consumption in operation, $\mathrm{W}$ \\
$p_{s}$ & Robot power consumption for sleeping, $\mathrm{W}$ \\
$d$ & Total trip distance to visit the energy nodes, $\mathrm{m}$ \\
$r$ & Robot speed, m/s \\
$T_{\text {dock }}$ & Average time to recognize and dock with an energy node, $\mathrm{s}$ \\
$E_{S}$ & Total energy storage capacity, Joule \\
$E_{\max }$ & Maximum energy obtained from the energy nodes, Joule \\
$R_{\max }$ & Robot energy storage capacity, Joule \\
\hline
\end{tabular}

We assume

- $\quad p_{\mathrm{s}}<g_{t o t}$, i.e., energy consumption rate in the sleep state is less than the total energy generation rate;

- $\quad p_{\mathrm{c}}<\eta \cdot \mu$, i.e., the energy gained by the robot during charging is greater than the energy consumed by the robot at rest; and

- robot energy storage capacity is greater than that for energy nodes.

Let $E(n)$ denote the energy the robot obtains from the energy nodes, and $R(n)$ denote robot energy level after the $n$-th charge cycle. And

$$
R(n)=R_{t o t}(n)-R_{o}
$$


where $R_{t o t}(n)$ is the total energy stored in the robot, and $R_{o}$ is the bottom energy, i.e., the minimum energy to ensure robot energy is not completely exhausted while moving to obtain energy, regardless of the order the nodes are visited. The time spent in a charge cycle is sum of robot working time, $t_{\text {work }}$, sleeping time, $t_{\text {sleep }}$, and time taken to acquire energy, $t_{\text {charge }}$. Hence, not considering energy saturation at nodes,

$$
E(n)=\eta \cdot\left(g_{\text {tot }} \cdot\left(t_{\text {work }}(n)+t_{\text {sleep }}(n)+t_{\text {charge }}(n)\right)-R_{s}(n)+R_{s}(n-1)\right),
$$

where $R_{S}(n)$ is the energy remaining in the energy nodes when the $n$-th charge cycle is completed, i.e., the obtained energy is the sum of the energy produced during one cycle and the energy remaining in the previous cycle minus the energy remaining in this cycle. Let $\tau_{n i}$ be the visit time for the $i$-th energy node in the $n$-th charge cycle, and $\tau_{n b}$ be the time to return to the base station after charging. Thus, energy remaining at the $i$-th node, $\varepsilon_{r i}$, and residual energy $R_{s}(n)$ are

$$
\varepsilon_{r i}(n)=g_{i} \cdot\left(\tau_{n b}-\tau_{n i}\right),
$$

and

$$
R_{s}(n)=\sum \varepsilon_{r i}(n)
$$

The time to get energy, $t_{\text {charge, }}$ is the sum of travel time between nodes, time to recognize and dock nodes, and time to receive energy,

$$
t_{\text {charge }}(n)=\frac{d}{r}+N \cdot T_{\text {dock }}+\frac{E(n)}{\eta \cdot \mu} .
$$

We can obtain $R(n)$ for the robot by subtracting energy consumed to obtain the energy from $E(n)$. The consumed energy is the sum of energy used moving toward the node and at rest during charging from Equation (4),

$$
\text { Consumed energy }=\left(\frac{d}{r}+N \cdot T_{\text {dock }}\right) \cdot\left(p_{m}+p_{c}\right)+\frac{E(n)}{\eta \cdot \mu} \cdot p_{c} .
$$

Suppose the $N$ energy nodes are uniformly distributed over a square area with length $D \mathrm{~m}$ of one side, and are visited following the Hamiltonian cycle. The distance to be traveled, $d$, can be approximated as [20]:

$$
0.625 D<\lim _{\mathrm{N} \rightarrow \infty} N^{-\frac{1}{2}} \cdot d<0.922 D
$$

\subsection{Survival Condition}

The survival condition for a robot with sleep function is that enough energy is produced during sleep time. On the other hand, robots without a sleep function must obtain more energy than they consume using only energy produced during working hours. If a robot with a sleep function is kept in a sleep state except for the time it takes to obtain energy, then

$$
t_{\text {work }}=0, t_{\text {sleep }}=R(n-1) / p_{s}
$$

whereas

$$
t_{\text {work }}=R(n-1) / p_{w}, t_{\text {sleep }}=0
$$

for a robot without sleep function. We approximate $R_{S}(n) \approx R_{S}(n-1)$ in Equation (6) to obtain a linear model, i.e., the energy remaining in the nodes is almost constant, and by rewriting Equations (6), (9), (10), (12) and (13),

$$
R(n)=A \cdot E(n)-B,
$$


and

$$
E(n)=C \cdot E(n-1)+D,
$$

where

$$
\begin{gathered}
A=1-\frac{p_{c}}{\eta \cdot \mu}, \\
B=\left(\frac{d}{r}+N \cdot T_{\text {dock }}\right) \cdot\left(p_{m}+p_{c}\right), \\
C=\eta \cdot g_{\text {tot }} \cdot \frac{A}{P_{*} \cdot\left(1-\frac{g_{\text {tot }}}{\mu}\right)^{\prime}}
\end{gathered}
$$

and

$$
D=\frac{\eta \cdot g_{\text {tot }}}{\left(1-\frac{g_{\text {tot }}}{\mu}\right)} \cdot\left(\frac{d}{r}+N \cdot T_{\text {dock }}-\frac{B}{P_{*}}\right),
$$

with $P^{*}=p_{\mathrm{s}}$ for a robot with sleep function and $P^{*}=p_{w}$ without. From the assumptions in Section 2.2, $A, B$ and $C>0$, and $D>0$ for robots with a sleep function. Thus, the solution for Equation (15) can be expressed as

$$
E(n)=\left(E(0)-\frac{D}{1-C}\right) C^{n}+\frac{D}{1-C},
$$

where $E(0)=(R(0)+B) / A$, and $R(0)$ is the initial robot energy.

Since energy node storage capacity is limited, total energy storage is saturated over time. Assuming that the storage amount of each node is proportional to the node energy generation rate, energy saturation for the entire node can be approximated by the saturation function. If $0<C<1$,

$$
E(n) \rightarrow \min \left(E_{\max }, \frac{D}{1-C}\right) .
$$

If $1 \leq C, E(n)$ reaches $E_{\max }$ and if energy obtained > energy consumed for a robot with a sleep function, then the extra energy can be used for work. When $R(n)<0$ in both cases, robot energy is depleted.

\subsection{Sleep Strategy}

A robot that does not have a sleep function needs to visit an energy node when its energy reaches zero while operating; whereas if the robot has a sleep function, it can enter a sleep state when energy production is insufficient. We present a sleep strategy to obtain a high utilization rate, $u$, i.e., the ratio of time spent working to the entire time,

$$
u=\frac{t_{\text {work }}}{t_{\text {work }}+t_{\text {sleep }}+t_{\text {charge }}} .
$$

The proposed sleep strategy is to obtain maximum energy per charge cycle with minimum sleep time. We assume the robot is under survival conditions with $C \geq 1$, hence extra energy is generated for work. The time to enter sleep state, $T_{\text {entry }}$, is obtained from robot energy after charging in the previous cycle, to obtain maximum energy, $E_{\max }$. If the energy level entering sleep state is $R_{\text {sleep }}$, then

$$
t_{\text {work }}=H\left(R(n-1)-R_{\text {sleep }}\right) \cdot\left(R(n-1)-R_{\text {sleep }}\right) / p_{w}
$$

and

$$
t_{\text {sleep }}=\min \left(R(n-1), R_{\text {sleep }}\right) / p_{s},
$$


where $H(\cdot)$ is a Heaviside step function. When charge and work are balanced, where $E_{e q}$ is balanced obtained energy, $R_{e q}$ is the balanced robot energy,

$$
\begin{gathered}
E_{e q}=\eta \cdot \operatorname{sat}_{E_{s}}\left(g_{\text {tot }} \cdot\left(t_{\text {work }}+t_{\text {sleep }}+t_{\text {charge }}\right)\right), \\
R_{e q}=A \cdot E_{e q}-B
\end{gathered}
$$

and hence,

$$
\begin{gathered}
t_{\text {work }}=H\left(R_{\text {eq }}-R_{\text {sleep }}\right) \cdot\left(R_{\text {eq }}-R_{\text {sleep }}\right) / p_{w}, \\
t_{\text {sleep }}=\min \left(R_{\text {eq }}, R_{\text {sleep }}\right) / p_{s},
\end{gathered}
$$

and

$$
t_{\text {charge }}=\frac{d}{r}+N \cdot T_{\text {dock }}+\frac{\mathrm{E}_{e q}}{\eta \cdot \mu} .
$$

This piecewise linear equation has solution $E_{e q}=E_{\max }$ when

$$
R_{\text {sleep }}=\left(E_{\text {max }} \cdot\left(\frac{1}{\eta \cdot g_{\text {tot }}}-\frac{A}{p_{w}}-\frac{1}{\eta \cdot \mu}\right)+\frac{B}{p_{w}}-\frac{d}{r}-N \cdot T_{\text {dock }}\right) /\left(\frac{1}{p_{s}}-\frac{1}{p_{w}}\right),
$$

and $R_{\text {sleep }} \geq 0$ and $R_{\text {sleep }} \leq E_{\text {max }} \cdot A-B$.

For minimum $R_{\text {sleep }}^{*}$ that satisfies Equation (26), we obtain $\mathrm{T}_{\text {entry, }}$, the sum of $t_{\text {work }}$ and $t_{\text {sleep }}$, as

$$
T_{\text {entry }}=H\left(R_{\text {eq }}-R_{\text {sleep }}^{*}\right) \cdot \frac{R_{\text {eq }}-R_{\text {sleep }}^{*}}{p_{w}}+\frac{\min \left(R_{\text {eq }}, R_{\text {sleep }}^{*}\right)}{p_{s}} .
$$

Thus, the proposed sleep strategy is:

Sleep Rule 1:

For elapsed time $t$ after starting work and current robot energy $R(t)$,

$1 \quad$ Work state $\rightarrow$ Sleep state

- If $t+R(t) / p_{s} \leq T_{\text {entry }}$, Enter sleep state

- else, maintain the work state

2 Sleep state $\rightarrow$ Charge state

- $\quad$ If $R(t) \leq 0$, Enter charge state

\section{Results}

\subsection{Numerical Evaluation}

\subsubsection{Finding Survival Area}

The survival area was obtained using the energy generation rate, $g_{t o t}$, and total travel distance as variables. The parameters used for numerical evaluation were $r=1 \mathrm{~m} / \mathrm{s}, T_{\text {dock }}=30 \mathrm{~s}, \mu=50 \mathrm{~W}, p_{c}=10$ $\mathrm{W}, p_{m}=20 \mathrm{~W}, p_{w}=25 \mathrm{~W}, p_{s}=2 \mathrm{~W}$, and $\eta=0.5$. Figure 4 shows the survival area for the robot from Equation (14) with respect to $g_{\text {tot }}$ and distance traveled to obtain energy. Figure $4 \mathrm{a}$,b show the survival area for the robot when total energy storage $=450$ and $2500 \mathrm{~kJ}$, respectively. 


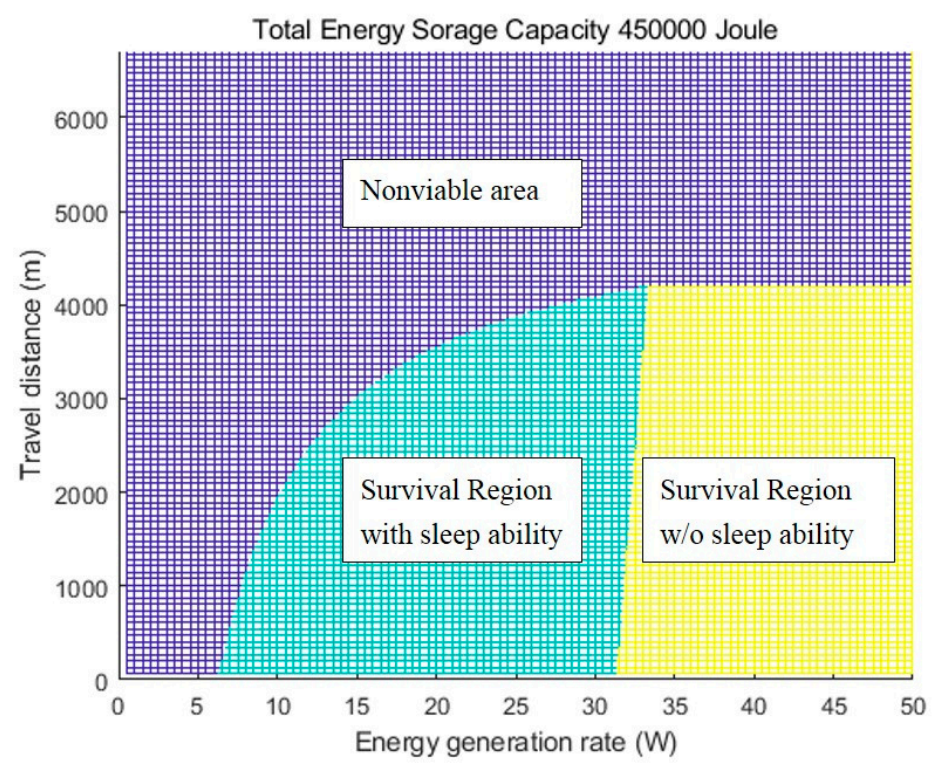

(a)

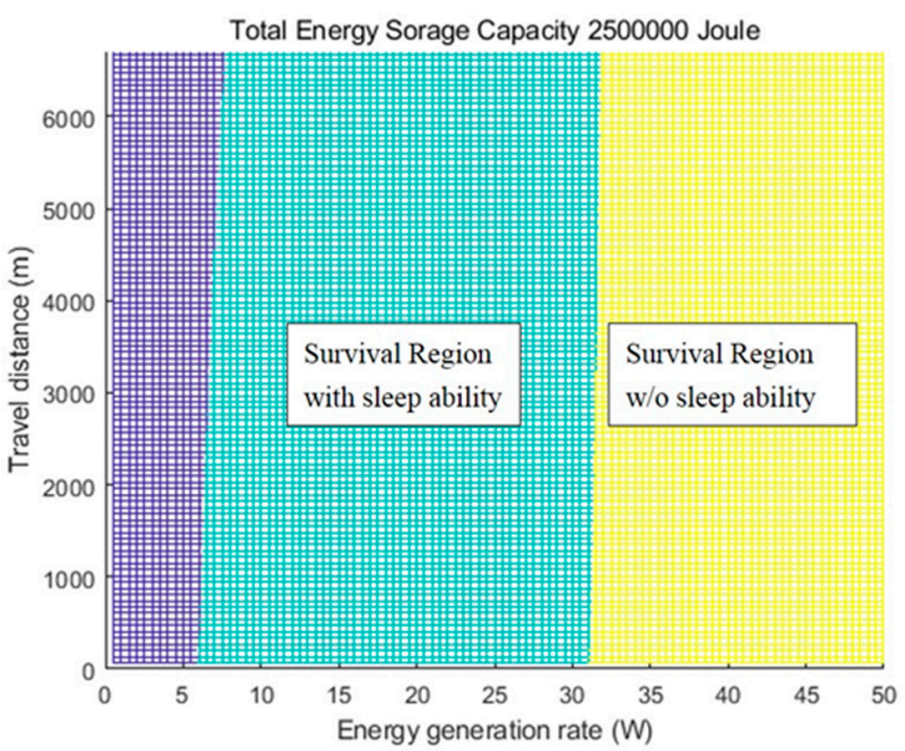

(b)

Figure 4. Robot survival region. (a) Total energy storage capacity $450 \mathrm{~kJ}$; (b) Total energy storage capacity $2500 \mathrm{~kJ}$.

\subsubsection{Simulation}

For the simulation study we set up 10 energy nodes in a $200 \mathrm{~m} \times 200 \mathrm{~m}$ area with total capacity $E_{S}=870 \mathrm{~kJ}$. The mobile robot visits nodes along the shortest distance Hamiltonian path, with total distance $=668.5 \mathrm{~m}$. Each energy node has a random energy generation rate and total energy generation rate $g_{t o t}=2,15$ and $35 \mathrm{~W}$, representing non-viable, viable with sleep function, and viable without sleep function, respectively. Figure 5 shows the robot energy stored with a sleep function after every recharge cycle. Figure 5a shows the amount of energy in the robot when the robot keeps the sleep state after charging. The robot became energy depleted for the non-viable condition. Figure $5 \mathrm{~b}$ shows the robot energy when the robot works after charging. The tasks performed by the robot consume $1-50 \mathrm{~W}, 25 \mathrm{~W}$ average, and working times are 10-60 s. Sleep state is entered if necessary according to the proposed sleep strategy during operation. 


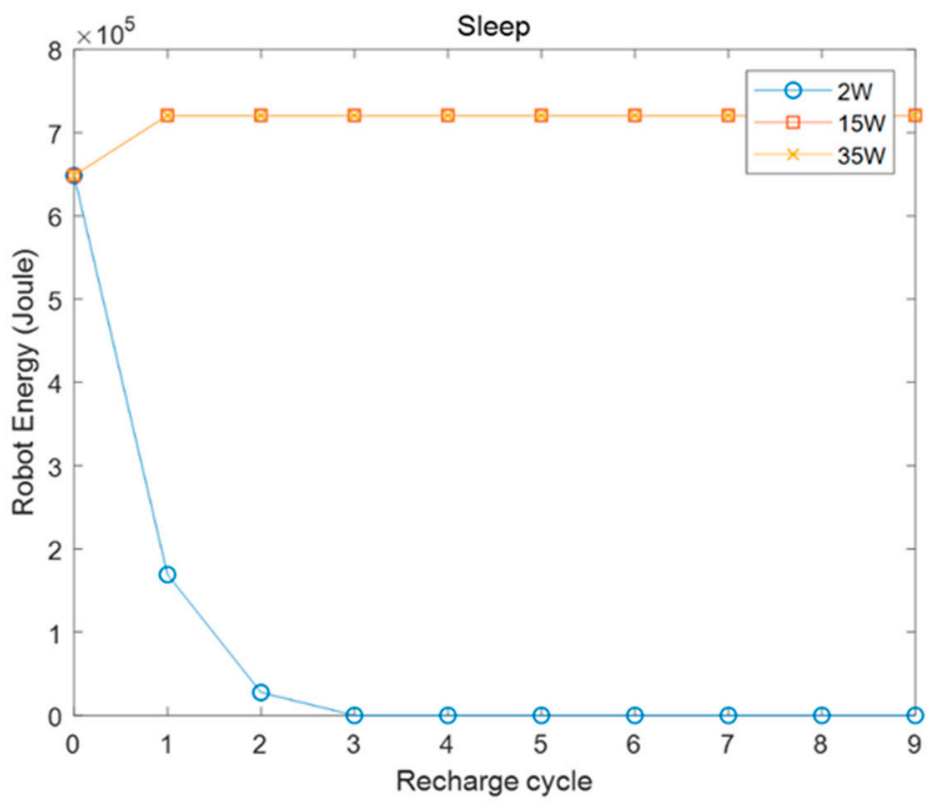

(a)

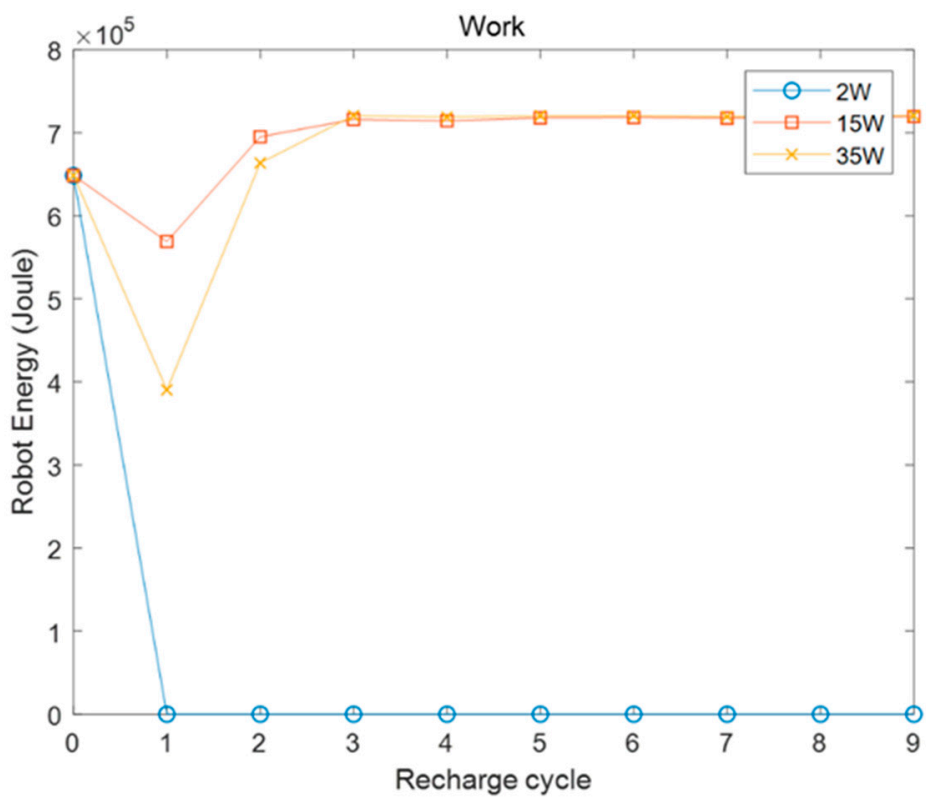

(b)

Figure 5. Robot energy after charge cycle. (a) Robot energy: sleep state maintained before charging; (b) Robot energy: work and sleep if necessary before charging.

Figure 6 shows the utilization rate for fixed energy and proposed sleep methods with an energy generation rate $=15$ and $35 \mathrm{~W}$. For the fixed energy-level sleep strategy, the robot enters sleep state when energy falls below the predetermined level $(130 \mathrm{~kJ})$. However, the proposed method has superior utilization. 


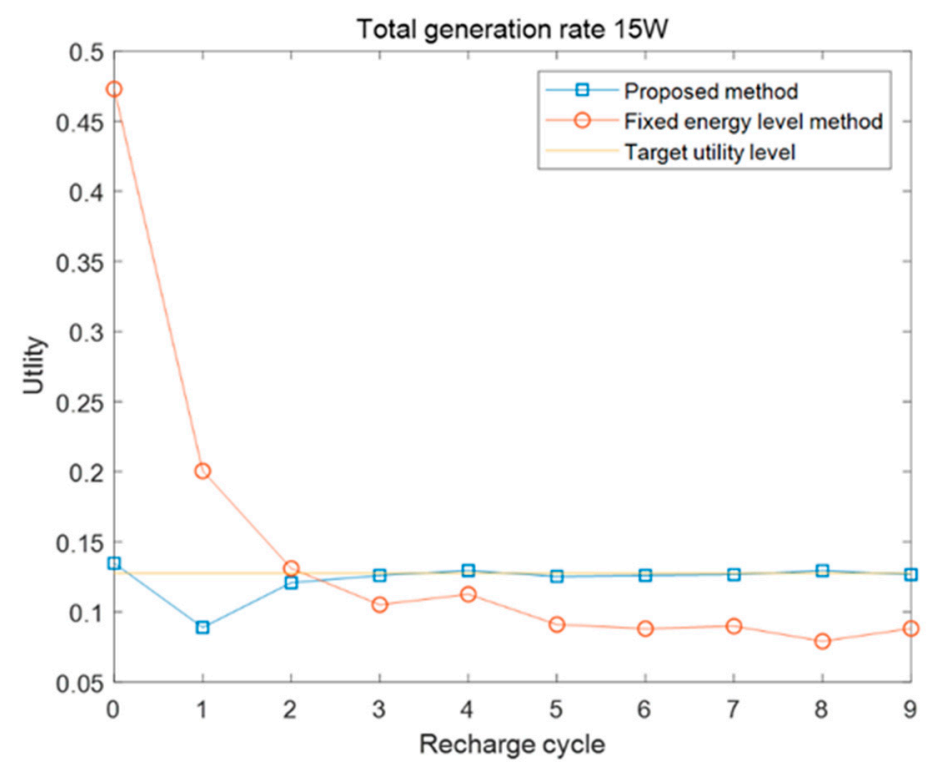

(a)

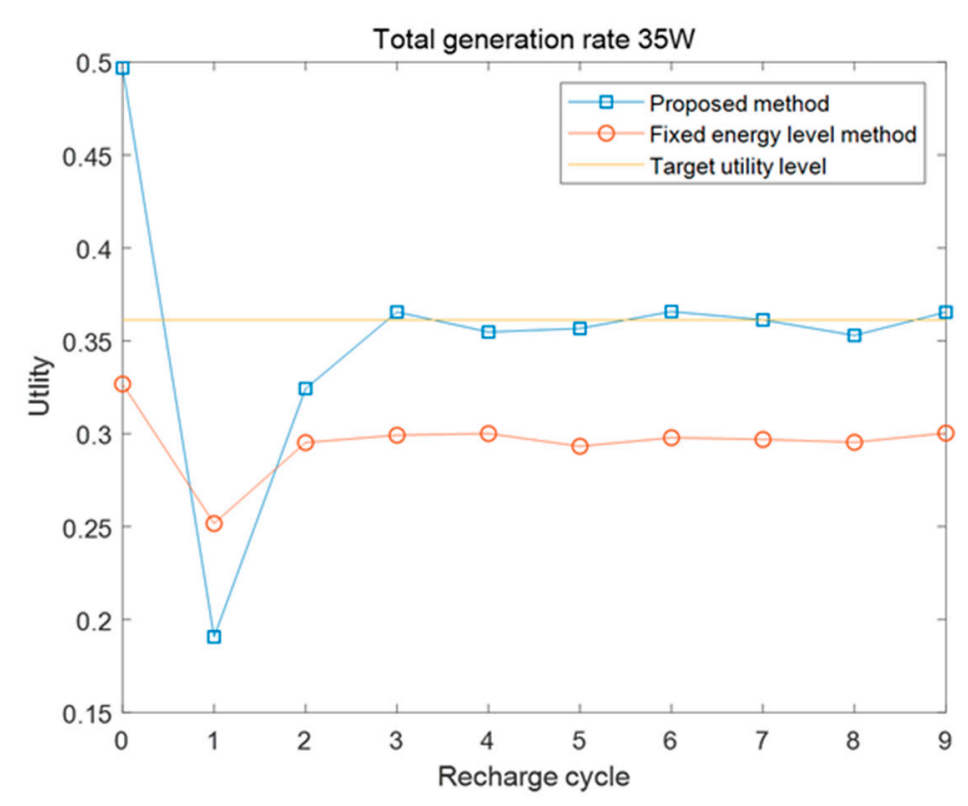

(b)

Figure 6. Mobile robot utilization rate. (a) Total generation rate $15 \mathrm{~W}$; (b) Total generation rate $35 \mathrm{~W}$.

\subsection{Experiments}

\subsubsection{Experimental Setup}

The puspose of the experiment was to verify the feasibility of the proposed energy nodes and energy transfer method. The experimental energy node comprised a thermoelectric module (TEM), generating electricity using waste heat. The robot knows the approximate position of the energy node, recognizes the node in that vicinity using image information, and aligns transmitting and receiving coils accurately. Figure 7 shows the thermoelectric power generation node. Electricity was generated 
by the Seebeck effect, where electromotive force is generated by temperature differences due to heating one side and radiating the other. The voltage generated is

$$
\mathrm{V}=\mathrm{M} \cdot \alpha \cdot\left(T_{H}-T_{C}\right),
$$

where $M$ is the number of modules, $\alpha$ is the Seebeck coefficient, and $T_{H}$ and $T_{C}$ are the hot and cool side temperatures, respectively [21].

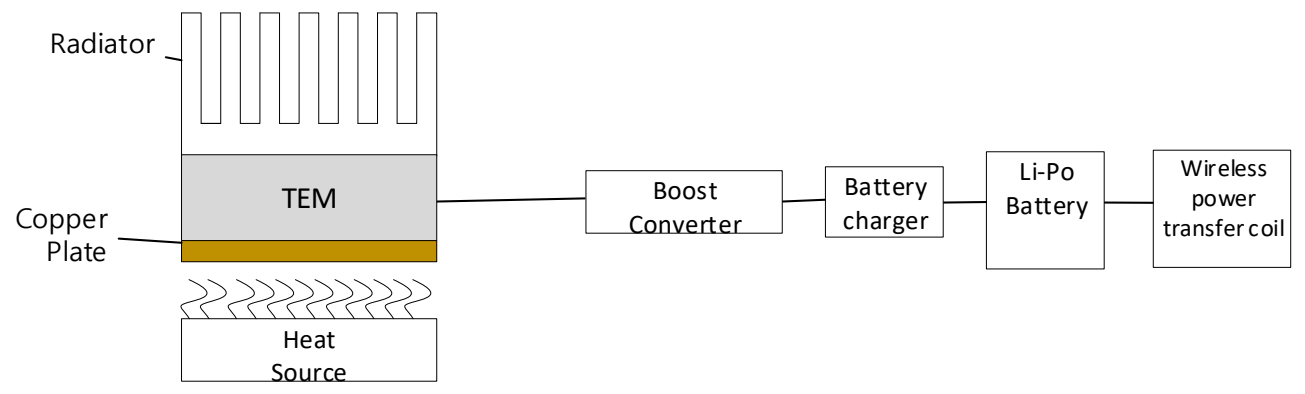

Figure 7. Thermoelectric energy nodule (TEM) structure.

Table 2 shows voltage, current, and power generated from the TEM used in this experiment. Considering the converter, charger, and battery efficiency, the overall energy generation rate is much lower than the TEM power.

Table 2. Thermoelectric energy module (TEM) outputs.

\begin{tabular}{ccccc}
\hline Condition & Voltage (V) & Current (A) & TEM Power (W) & $\begin{array}{c}\text { Estimated Available } \\
\text { Power (W) }\end{array}$ \\
\hline$\Delta \mathrm{T}=\mathbf{4 7}{ }^{\circ} \mathrm{C}\left(\boldsymbol{T}_{\boldsymbol{H}}=\mathbf{1 6 8}{ }^{\circ} \mathrm{C}\right)$ & 10.34 & 0.49 & 5.07 & 2.6 \\
\hline$\Delta \mathrm{T}=\mathbf{6 4}^{\circ} \mathrm{C}\left(\boldsymbol{T}_{\boldsymbol{H}}=\mathbf{1 6 8}{ }^{\circ} \mathrm{C}\right)$ & 13.61 & 0.66 & 8.98 & 4.6 \\
\hline
\end{tabular}

The robot used in the experiment was Turtlebot3, which is widely used as an experimental mobile robot platform, and had a laser scanner and a monocular camera for navigation and object recognition. Robot navigation techniques based on laser scanners can be found in Reference [22]. The mobile robot consumed $4.2 \mathrm{~W}$ at rest, due to the controller, and travelled flat terrain at $0.2 \mathrm{~m} / \mathrm{s}$, consuming an additional $5.9 \mathrm{~W}$. The wireless power transfer module used in the experiment was a commercial device that conforms to the Qi standard and theoretical maximum energy transfer rate $\mu=15 \mathrm{~W}$. Thus, for average $p_{w}=8 \mathrm{~W}, p_{s}=2 \mathrm{~W}, \eta=0.5$, and energy acquisition area $=2500 \mathrm{~m}^{2}$, the robot with a sleep function was expected to survive with three energy nodes with specifications as above. The wireless power transfer module required lateral position accuracy $=1 \mathrm{~cm}$ for efficiency [17]. After moving to the charging station vicinity, the robot recognized the alignment marker from images, detected four points on the marker, and calculated the position and direction for the correct charging coil, as shown in Figure 8. 


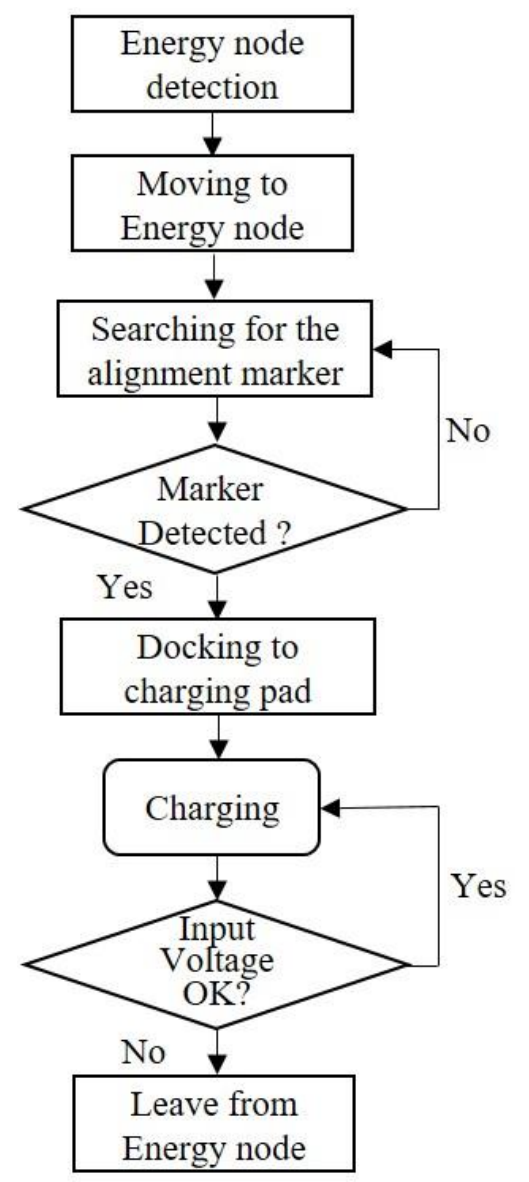

Figure 8. Robot charging process for the experiment.

\subsubsection{Experimental Results}

The robot was initially near an energy node, and then detected it specifically. The robot learned the energy node shape in advance using the single shot detector (SSD) algorithm [23]. Figure 9 shows recognition and identification results for two energy node types. The detection of the object was performed in about $1 \mathrm{~s}$ with a $1.2 \mathrm{GHz}$ embedded robot controller. Figure 10 shows that the robot then estimated the position and direction of the energy node and the predetermined transmission coil pad. The transmitting and receiving coils must be accurately aligned to ensure a high transmission efficiency. Robot position and direction were corrected by recognizing the marker ahead of the charge station, as shown in Figure 11, along with the estimated relative pose. Figure 12 shows the final docking with the charging coil, confirming the position accuracy required for wireless charging was obtained using the image information. 


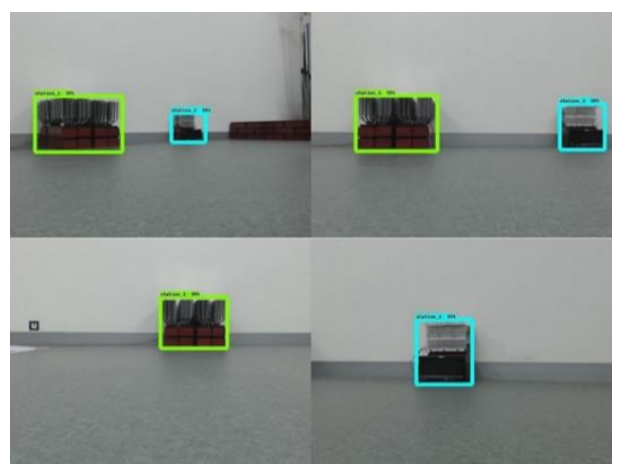

Figure 9. Energy node detection.

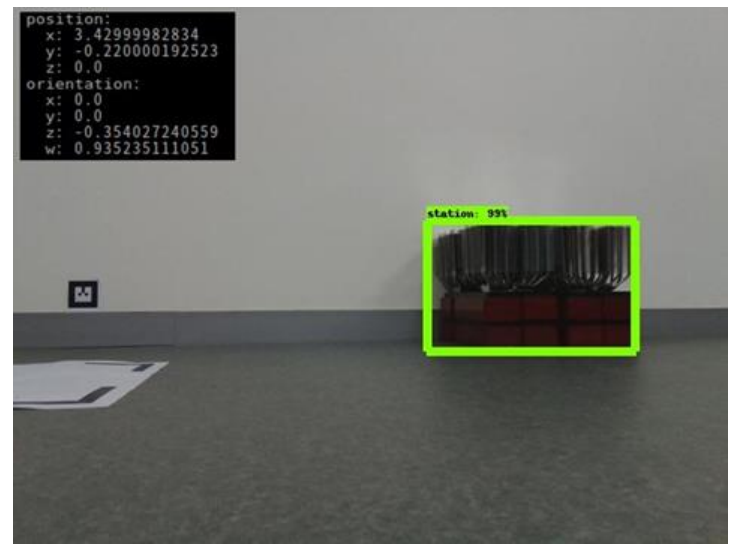

Figure 10. Energy node location and orientation estimation.

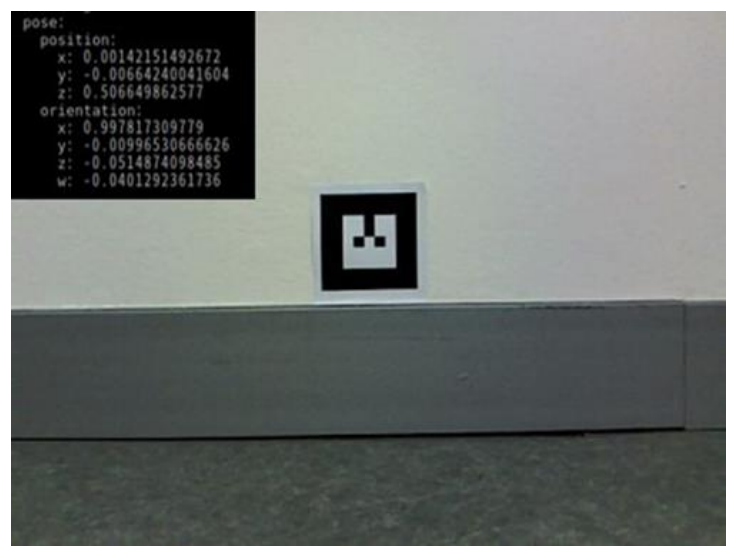

Figure 11. Coil alignment marker detection. 


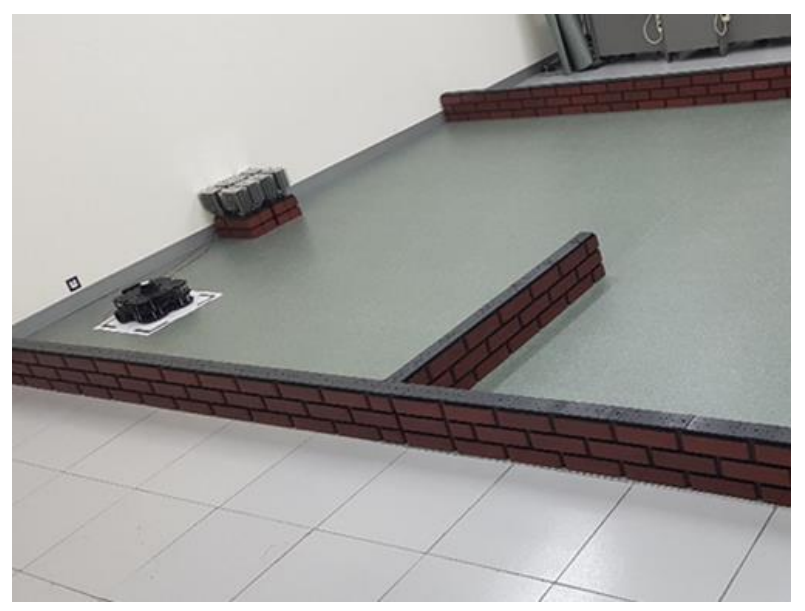

(a)

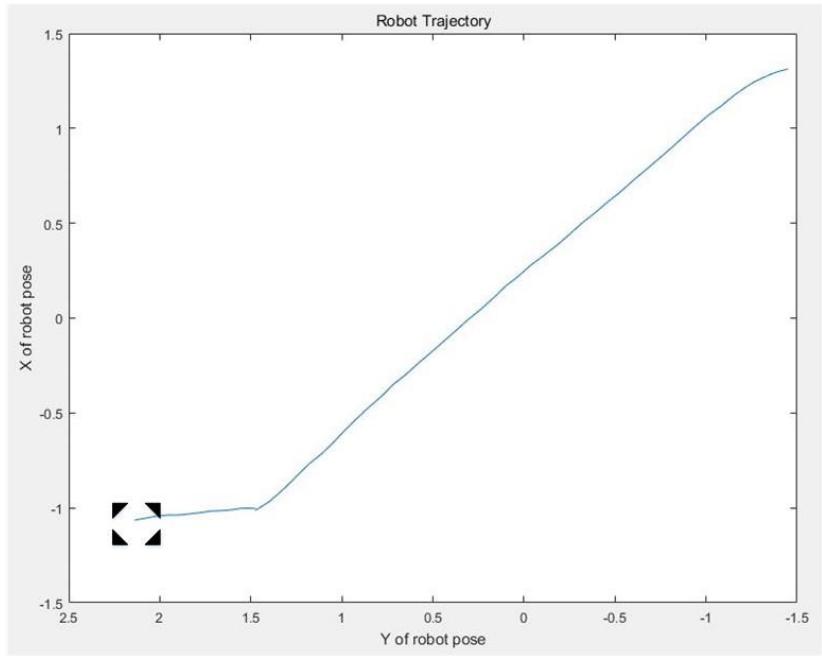

(b)

Figure 12. Docking with the charging coil. (a) Robot on charging pad; (b) Robot trajectory.

\section{Discussion}

This study proposed a robot system where a mobile robot obtained energy from small-scale energy sources distributed over a large area, e.g., as might be expected for renewable energy sources. Electric energy produced by the small-scale generators were stored in the battery and then transferred to the robot. The charging process was modeled as a linearized difference equation to obtain robot survival conditions, and we adopted wireless charging to solve the mechanical complexity for energy transfer using contact charging. Previous robot energy autonomy studies [8,9] have only considered basic operations, transferring generated energy directly to the robot and the energy balance was not considered. In contrast, in this study we formulated conditions such that the robot could balance work and charge using parameters representing a node geometric distribution, energy generation rate, and energy storage capacity. We implemented a compact energy node comprising thermoelectric elements to verify the feasibility of the proposed system and implemented an auto charge test. Energy nodes can have various physical forms depending on how energy is generated, so the robot's ability to recognize an energy source is essential. The robot recognized the energy nodes stably using the SSD algorithm, and image information was then used to accurately align the robot and transmitting coil. The robot achieved sufficient precision for wireless charging using a simple alignment marker, confirming they would be suitable for robot energy autonomy.

Several practical problems remain to be addressed. 
- The current absence of a specialized energy transfer device for robot charge means the charging rate is slow for practical use.

- Battery efficiency deteriorates with extended charge-discharge cycles, and it is difficult to know how much available energy remains within the battery.

Ideally, these problems should be solved, but any realistic situation requires a conservative design.

Author Contributions: Hardware implementation, J.K.; experiment, J.K.; conceptualization, C.M.; writing, C.M.

Funding: This research was supported by the Basic Science Research Program through the National Research Foundation of Korea (NRF) funded by the Ministry of Education (2017R1D1A1B03031245).

Acknowledgments: This research was supported by the Basic Science Research Program through the National Research Foundation of Korea (NRF) funded by the Ministry of Education (2017R1D1A1B03031245).

Conflicts of Interest: The authors declare no conflict of interest.

\section{References}

1. Kartoun, U.; Stern, H.; Edan, Y.; Feied, C.; Handler, J.; Smoth, M.; Gillam, M. Vision-Based Autonomous Robot Self-Docking and Recharging. In Proceedings of the World Automation Congress, Budapest, Hungary, 24-26 July 2006; pp. 1-8. [CrossRef]

2. Koo, K.M.; Shin, K.C. Apparatus for Automatic Charging of the Autonomous Mobile Robot and Method for Automatic Charging Used the Same. Patent US20080174268A1, 24 July 2008.

3. Park, S.; Lee, Y.-J. Entertainment Walking Robot that Feeds on Batteries. In Proceedings of the IEEE/RSJ International Conference on Intelligent Robots and Systems, San Diego, CA, USA, 29 October-2 November 2007; pp. 2035-2040. [CrossRef]

4. Wu, Y.-C.; Teng, M.-C.; Tsai, Y.-T. Robot Docking Station for Automatic Battery Exchanging and Charging. In Proceedings of the IEEE International Conference on Robotics and Biomimetics, Guilin, China, 13-19 December 2009; pp. 1043-1046. [CrossRef]

5. Melhuish, C.; Ieropoulos, I.; Greenman, J.; Horsfield, I. Energetically autonomous robots: Food for thought. Auton. Robot. 2006, 21, 187-198. [CrossRef]

6. Abbott, J.J.; Nagy, Z.; Beyeler, F.; Nelson, B.J. Robotics in the Small. IEEE Robot. Autom. Mag. 2007, 14, 92-103. [CrossRef]

7. Rae, C.; Bradley, F. Energy autonomy in sustainable communities-A review of key issues. Renew. Sustain. Energy Rev. 2012, 16, 6497-6506. [CrossRef]

8. Ngo, T.D.; Raposo, H.; Schiøler, H. Potentially Distributable Energy: Towards Energy Autonomy in Large Population of Mobile Robots. In Proceedings of the 2007 IEEE International Symposium on Computational Intelligence in Robotics and Automation, Jacksonville, FL, USA, 20-23 June 2007. [CrossRef]

9. Khonji, M.; Alshehhi, M.; Tseng, C.-M.; Chau, C.-K. Autonomous Inductive Charging System for Battery-operated Electric Drones. In Proceedings of the Eighth International Conference on Future Energy Systems, Karlsruhe, Germany, 12-15 June 2017; pp. 322-327. [CrossRef]

10. Shinohara, N. Wireless Power Transfer; IET: Croydon, UK, 2018.

11. Ju, H.; Zhang, R. Throughput maximization in wireless powered communication networks. IEEE Trans. Wirel. Commun. 2014, 13, 418-428. [CrossRef]

12. Vamvakas, P.; Tsiropoulou, E.; Vomvas, M.; Papavassiliou, S. Adaptive power management in wireless powered communication networks: A user-centric approach. In Proceedings of the 2017 IEEE 38th Sarnoff Symposium, Newark, NJ, USA, 19-20 September 2017; pp. 1-6. [CrossRef]

13. Kim, J.; Rho, S.; Moon, C.; Ahn, H. Imaging Processing based a Wireless Charging System with a mobile Robot. In Computer Applications for Database, Education, and Ubiquitous Computing, Communications in Computer and Information Science; Springer: Berlin/Heidelberg, Germany, 2012; Volume 352, pp. 298-301. [CrossRef]

14. Cortes, I.; Kim, W.-J. Autonomous Positioning of a Mobile Robot for Wireless Charging Using Computer Vision and Misalignment-Sensing Coils. In Proceedings of the Annual American Control Conference, Milwaukee, WI, USA, 27-28 June 2018; pp. 4324-4329. [CrossRef]

15. Junaid, A.B.; Konoiko, A.; Zweiri, Y.; Sahinkaya, M.N.; Seneviratne, L. Autonomous Wireless Self-Charging for Multi-Rotor Unmanned Aerial Vehicles. Energies 2017, 10, 803. [CrossRef] 
16. Peng, Y.; Li, Z.; Zhang, W.; Qiao, D. Prolonging Sensor Network Lifetime Through Wireless Charging. In Proceedings of the 31st IEEE Real-Time Systems Symposium, San Diego, CA, USA, 30 November-3 December 2010. [CrossRef]

17. Kim, J.-O.; Moon, C. A Vision-Based Wireless Charging System for Robot Trophallaxis. Int. J. Adv. Robot. Syst. 2015, 12, 177. [CrossRef]

18. Rahn, C.D.; Wang, C.-Y. Battery Systems Engineering; Wiley: Hoboken, NJ, USA, 2013.

19. Mei, Y.; Lu, Y.-H.; Hu, Y.C.; Lee, C.S.G. A Case Study of Mobile Robot's Energy Consumption and Conservation Techniques. In Proceedings of the 12th International Conference on Advanced Robotics, Seattle, WA, USA, 17-20 July 2005; pp. 492-497. [CrossRef]

20. Steinerberger, S. New Bounds for the Traveling Salesman Constant. Adv. Appl. Probab. 2013, 47, 27-36. [CrossRef]

21. Bell, L.E. Cooling, Heating, Generating Power, and Recovering Waste Heat with Thermoelectric Systems. Science 2008, 321, 1457-1461. [CrossRef] [PubMed]

22. Castellanos, J.; Tardss, J.; Tardos, J. Mobile Robot Localization and Map Building. Springer: New York, NY, USA, 2000.

23. Shanmugamani, R. Deep Learning for Computer Vision; Packt: Birmingham, UK, 2018.

(C) 2019 by the authors. Licensee MDPI, Basel, Switzerland. This article is an open access article distributed under the terms and conditions of the Creative Commons Attribution (CC BY) license (http://creativecommons.org/licenses/by/4.0/). 